\title{
Emerging technologies for management of patients with amyotrophic lateral sclerosis: from telehealth to assistive robotics and neural interfaces
}

\author{
Raffaele Pugliese $^{1}\left[\right.$ D $\cdot$ Riccardo Sala $^{1} \cdot$ Stefano Regondi ${ }^{1,2} \cdot$ Benedetta Beltrami $^{1} \cdot$ Christian Lunetta $^{1,2}$
}

Received: 17 December 2021 / Revised: 11 January 2022 / Accepted: 12 January 2022 / Published online: 21 January 2022

(c) The Author(s), under exclusive licence to Springer-Verlag GmbH Germany 2022

\begin{abstract}
Amyotrophic lateral sclerosis (ALS), also known as motor neuron disease, is characterized by the degeneration of both upper and lower motor neurons, which leads to muscle weakness and subsequently paralysis. It begins subtly with focal weakness but spreads relentlessly to involve most muscles, thus proving to be effectively incurable. Typically, death due to respiratory paralysis occurs in 3-5 years. To date, it has been shown that the management of ALS patients is best achieved with a multidisciplinary approach, and with the help of emerging technologies ranging from multidisciplinary teleconsults (for monitoring the dysphagia, respiratory function, and nutritional status) to brain-computer interfaces and eye tracking for alternative augmentative communication, until robotics, it may increase effectiveness. The COVID-19 pandemic created a spasmodic need to accelerate the development and implementation of such technologies in clinical practice, to improve the daily lives of both ALS patients and caregivers. However, despite the remarkable strides that have been made in the field, there are still issues to be addressed. This review will be discussed on the eureka moment of emerging technologies for ALS, used as a blueprint not only for neurodegenerative diseases, examining the current technologies already in place or being evaluated, highlighting the pros and cons for future clinical applications.
\end{abstract}

Keywords Amyotrophic lateral sclerosis · Motor neuron diseases · Clinical management $\cdot$ Emerging technologies · Telemedicine $\cdot$ Brain-computer interface $\cdot$ Eye tracking $\cdot$ Robotics $\cdot$ Internet of things

\section{Introduction}

Amyotrophic Lateral Sclerosis (ALS) is a rapid progressive neurodegenerative disease characterized by the degeneration of both upper and lower motor neurons, leading to motor and extra-motor symptoms with death usually occurring 3-5 years after the onset of symptoms $[1,2]$. Recent studies suggest a worldwide incidence of $2 / 100,000$ individuals with ALS per year and a prevalence of 3-8 cases per 100,000 inhabitants [3]. The early detection of ALS can vary between patients: some present with spinal-onset

Raffaele Pugliese

raffaele.pugliese@nemolab.it

$\triangle$ Christian Lunetta

chrisitan.lunetta@nemolab.it

1 NeMO Lab, ASST Niguarda Cà Granda Hospital, Milan, Italy

2 NEuroMuscolar Omnicentre, Milan, Italy disease characterized by muscle weakness of the limbs, other patients present with bulbar-onset disease, which is characterized by dysarthria and dysphagia [4]. Although the primary symptoms of ALS are associated with motor dysfunction (i.e. muscle weakness, spasticity, and dysphagia), up to $50 \%$ of patients develop cognitive and/or behavioral impairment during the course of disease, and $13 \%$ of patients present with concomitant behavioral variant frontotemporal dementia (FTD) [5, 6]. Anyhow, loss of respiratory muscle innervation and associated complications are the most frequent causes of death [7]. The mechanism of neurodegeneration in ALS is not entirely clear yet (there are several cellular and molecular processes that are implicated) [8,9], and despite the continuous efforts to develop curative therapies, to date there is no effective cure to reverse the disease except for Riluzole [10, 11], Edaravone [12, 13], and non-invasive ventilation (NIV) $[14,15]$ that can improve quality of life and survival in ALS patients.

Currently, the main effort that is made regarding this pathology concerns patient care management, which 
includes extensive symptomatic and support therapies throughout the course of the disease and requires constant counseling to the patient and caregiver. Indeed, the current practice of caring for patients with ALS involves a multidisciplinary team consisting of neurologist, pulmonologist, physiatrist, nurse, physical and occupational therapists, speech pathologist, dietitian, social worker, mental health counselor, and respiratory therapist $[16,17]$. This type of care aims to optimize patients' quality of life and survival. For this reason, patients must be monitored closely and have continuous access to multidisciplinary centers during their disease. However, many ALS patients have access issues in the multidisciplinary clinics-mostly related to long travel distances, difficulty traveling, and long days at the clinic - and, in addition, there is a lack of monitoring patients between clinic visits. These critical issues could limit the continuity of multidisciplinary management of ALS patients, negatively affecting the course of the disease.

In this context, emerging technologies such as teleconsults, Internet of things (IoT), wearable devices, augmentative and assistive communication with brain-computer interfaces (BCI) and eye tracking (ET) support, and robotic rehabilitation can fill this gap [18-20] (Fig. 1). Such technologies could allow a specialist team to care for patients throughout their disease regardless of patients' ability to travel to the multidisciplinary centers. It should be also emphasized that the COVID-19 pandemic has rushed the need to remotely continue providing the best care to patients, therefore diminishing the risk for nosocomial infection during hospitalization [21]. However, to be successful and effective these emerging technologies must be accessible to all patients who may be frail, have severe disabilities, or have communication difficulties, and must be user-friendly to obtain better outcomes. Furthermore, to have a widespread and consolidated adaptation of these technologies-starting from the early stages of the disease-it is necessary to ensure that either ALS patients or caregivers acquire considerable control and independence in their use. Hence, it will be necessary to keep in mind that young, educated and professionally active patients will be more sensitive to emerging technologies, while older patients, not previously trained about such technologies, will face considerable difficulties, requiring more time, encouragement, and greater support by specialists from multidisciplinary centers.

Here will be evaluated the potential benefits and challenges of using emerging technologies (already in place or under evaluation) to facilitate care access and to improve the daily living of both ALS patients and caregivers.

\section{A Eureka moment to emerging technologies: matters and pitfalls}

There is considerable and growing interest in emerging technologies, especially from the social, economic, industrial, healthcare, and policy perspective [22, 23]. The evidence of the increasing attention being paid to the phenomenon of emerging technologies is clearly visible in the growing number of publications dealing with this topic, as depicted in Fig. 2. In the last two decades, there has been an exponential growth in publications focused on emerging technologies in general, with a total of 18,856 publications, as well as on emerging technologies for healthcare applications (1919 publications).

However, to date, there is a lack of consensus-among the scientometric community-on the definition of emerging technology [24]. To the best of our knowledge, Rotolo et al., have attempted to contribute to this ongoing debate through the conceptual clarification of the emerging technology phenomenon [25]. They identified five attributes that characterize emerging technologies: (1) radical novelty, (2) relatively fast growth, (3) coherence, (4) prominent impact, and (5) uncertainty and ambiguity. Furthermore, the authors defined emerging technologies as: "a relatively fast-growing
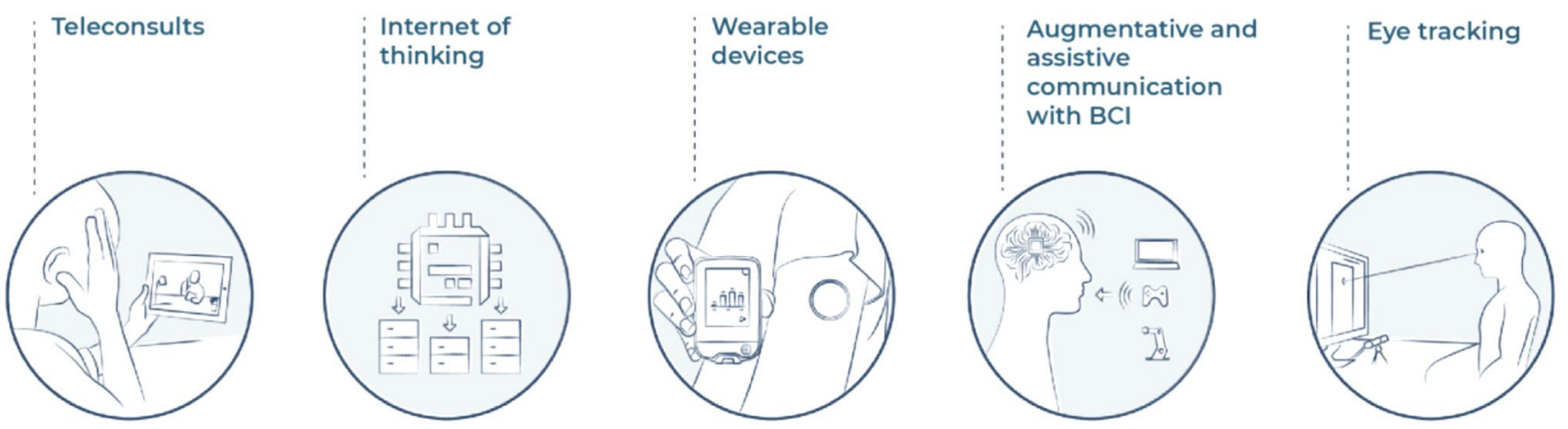

Fig. 1 Schematic representation of emerging technologies already used for the management of ALS patients 


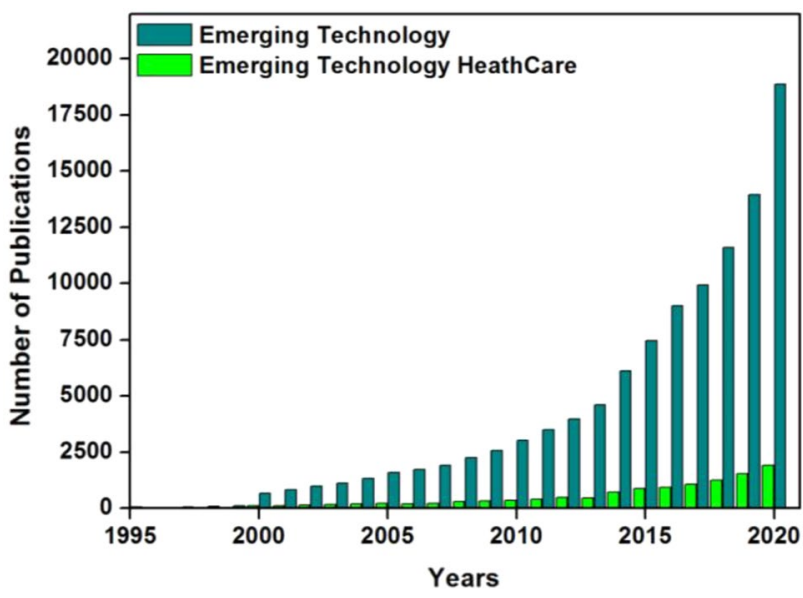

Fig. 2 Timeline of publications dealing with the topic of emerging technologies (in dark green) and emerging technologies in healthcare (light green) retrieved by querying PubMed. As inclusion criteria for the search on said database, the following key terms were used: "emerg* technol*" or "emerg* technol* in healthcare"

and radically novel technology characterized by a certain degree of coherence persisting over time and with the potential to exert a considerable impact on the socio-economic domains which is observed in terms of the composition of actors, institutions and the patterns of interactions among those, along with the associated knowledge production processes. Its most prominent impact, however, lies in the future and so in the emergence phase is still somewhat uncertain and ambiguous" [25].

We foresee a number of great opportunities for future research in this field, especially for the home management of patients with neurodegenerative, and cardiovascular diseases. First, future research should pay more attention to the origins of emerging technologies and should involve specialists from different fields such as engineers, physicians, biotechnologists, data scientists, and physicists (to name a few), to limit the risk factors and get better results in a short time. Second, the growing access to big data could help in the rationale of research on emerging technologies by delineating also the counterfactual samples (if present), thus providing a significant opportunity to develop indicators and methods for the evaluation and feasibility of emerging technologies, for which the current state of the art provides only a limited contribution. Finally, regarding the use of emerging technologies for the management of patients with neurodegenerative diseases such as ALS, spinal cord injuries (SCI), Alzheimer's, and Parkinson's, these should broaden their horizons not only on diagnostics but also on assessment, monitoring, prevention, education, consultation, and coaching, with the ultimate goal of transforming healthcare by moving it from hospitals/clinics to home care.

\section{Telemedicine: a breakthrough on predictive, personalized, preventive and participatory (4P) medicine}

Telemedicine is a general term, first introduced in the 1970s, to indicate the use of telecommunications technology to provide health care services to persons who are at some distance from the healthcare professional provider [26]. Analogous to the long-established physician-patient relationship, telemedicine must comply with all the rights and duties of any health act for prevention, diagnosis, treatment, rehabilitation, and monitoring. Furthermore, telemedicine must not replace traditional health services but rather to integrate them to improve effectiveness. The COVID-19 pandemic has accelerated this process and forced researchers and clinicians to reshape the telehealth strategies with the use of emerging technologies, and to accelerate its development for home care purposes. Indeed, the healthcare systems have suddenly faced an enormous and complete rearrangement of resources and spaces, and most visits for chronic diseases have been canceled or postponed. In this context, patients affected by chronic neurological diseases, such as ALS, are at risk of being lost at follow-up with a consequently higher morbidity and mortality.

Therefore, it is important to establish a well-structured telemedicine service to replace face-to-face visits, monitor disease's progression, and manage complications like life-threatening ones.

In this context, the American Academy of Neurology published some advice for improving a telemedicine service and suggested tools to perform a neurological examination remotely [27].

Luis Garcia-Gancedo et al. [28] reported a pilot observational study to investigate the feasibility of a digital platform for remote data collection of multiple symptoms, such as physical activity, heart rate variability, and digital speech characteristics, in 25 patients with ALS over 48 weeks (mean age of the overall population is: $53.1 \pm 9.93$ ). Such platform comprised three main components: (1) the commercially available Mega Faron 180 accelerometer and 2-lead ECG sensor, attached to the chest; (2) a Life-Insight Hub, that received data from the sensor via a secure Bluetooth wireless signal every $2 \mathrm{~min}$, and which in turn automatically uploaded data in real time to secure cloud servers; (3) a digital speech capture system comprising a high-fidelity microphone connected to a computer, with bespoke software that instructed the patients to say a series of vowels, words, and paragraphs, which were then recorded and immediately automatically transferred to a secure server via mobile connectivity (Fig. 3).

Overall, the authors reported that the amount and quality of the data collected and the accuracy of the algorithms 


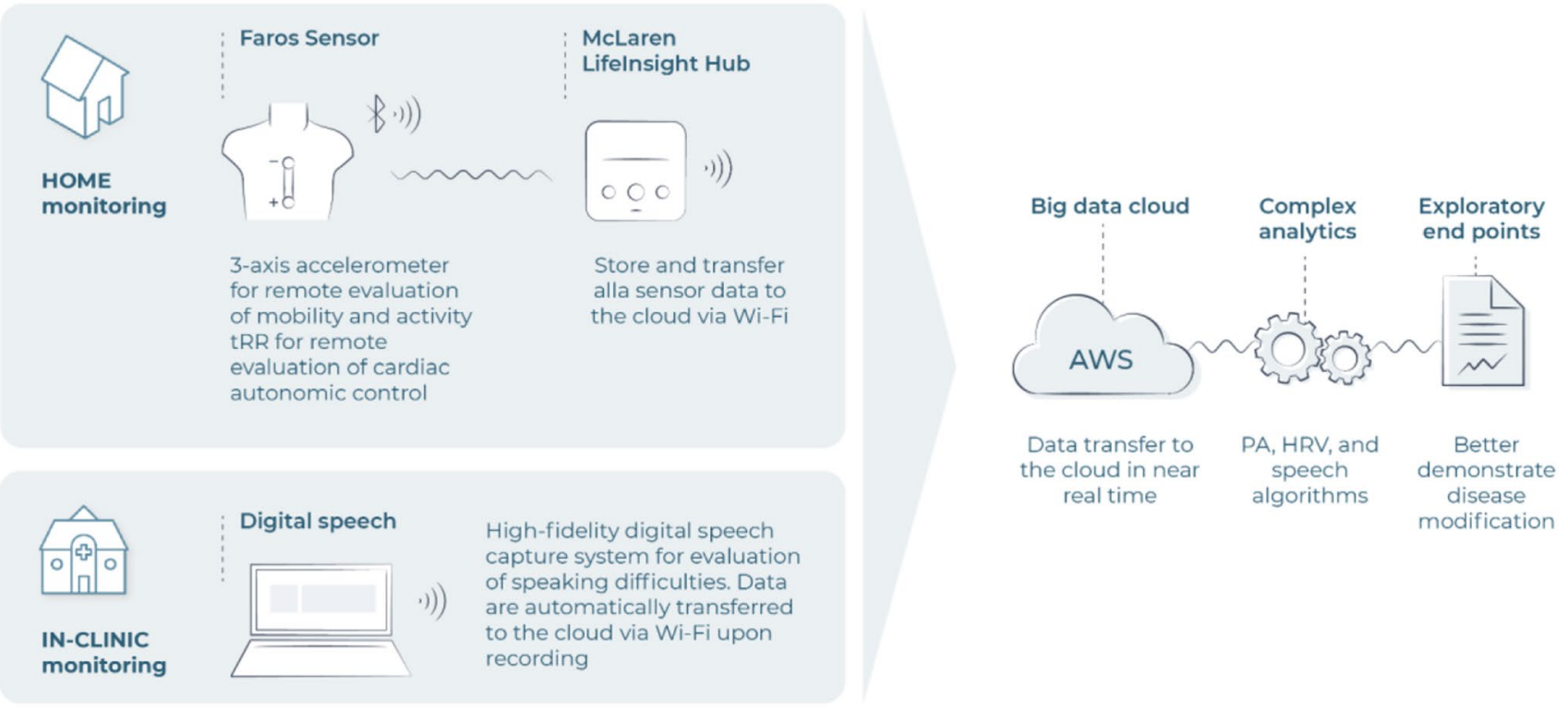

Fig. 3 Schematic representation of the monitoring platform reported by Luis Garcia-Gancedo et al. which comprises: Mega Faron 180, LifeInsight Hub, and a digital speech capture system

developed were sufficient to evaluate changes over time in patients' activities of daily living. In addition, the equipment was generally well tolerated by patients with no or minimal impact on their daily activities. Although, 6 of 25 patients had mild or moderate adverse events in the skin and subcutaneous tissue disorders category caused by the electrode patch. A limitation of this study-as specified by the authors themselves-was a lower than expected reception on the amount of home monitoring physical and speech activity data, partly because of a number of patients withdrawing from the study early, which limited the ability to draw robust clinical conclusions, and partly due to poor contact between the sensor and the skin.

Instead, other studies have been conducted to assess the feasibility and acceptability of telemedicine for treating ALS via real-time videoconferencing from the clinic to patients' homes [29-34]. Globally, these studies (one of which conducted in Italy during the COVID-19 pandemic) [31] found that patients, caregivers, and clinical staff members are satisfied with the telemedicine service, although improvements in technology and methods are needed to provide satisfactory person-to-person contactless remote assistance (Table 1).

Another key issue to be addressed in ALS patients is the monitoring of the nutritional status, which is a relevant prognostic factor [32]. Despite countless nutrition-based mobile health applications being available, only a few were clinically assessed [35-37]. In a recent randomized trial, Anne Marie Wills et al., compared remote nutritional counseling with or without mobile health technology in ALS patients [35]. In this randomized clinical trial, patients were randomly assigned one of three nutritional interventions, namely: (1) counseling by their physician or nurse ("standard care"); (2) counseling by a registered
Table 1 Advantages, disadvantages, and possible improvements experienced by the patient, caregivers, and clinical staff during the pilot studies

\begin{tabular}{lll}
\hline Advantages & Disadvantages & Improvements \\
\hline No travel & No personal touch & Better appointment options \\
In home & No emotional connection & Additional software options \\
Less time & Poor eye contact & Limit to necessary providers \\
Less effort & Video/audio & Better coordination of onsite support \\
No bad weather & Wait time between providers & Better video/audio \\
More convenient & Privacy & \\
Less stress & Not leaving house & \\
Saw clinical staff & Coordination with onsite support & \\
No overnight stay & No physical examination & \\
No childcare worries & & \\
\hline
\end{tabular}


dietitian ("in-person"); (3) counseling supported by a nutrition-based application ("mHealth"). All nutritional interventions received tailored nutrition recommendations and recorded dietary intake and weight biweekly (for mHealth) or monthly (for in-person). Moreover, based on patients' gain or loss weight, the nutritionist could remotely access the app and modify dietary recommendations. The primary outcome of weight and secondary and tertiary outcomes of calorie intake, ALS Functional Rating Scale Revised (ALSFRS-R), and quality of life were recorded at each clinic visit and analyzed in an intent-totreat (ITT) mixed model analysis.

Interestingly, patients with mHealth app-supported counseling increased their dietary intake and percent of recommended calories compared to patients who received standard-of-care treatment. Furthermore, the rate of disease progression measured by ALSFRS-R total score over 6 months, was half as fast among mHealth participants compared to participants in the standard care.

Based on these assumptions, in our opinion, Telemedicine in ALS is poised to monitor everyday life and potential life-threatening aspects of the disease, while creating new challenges and opportunities without replacing the traditional clinical procedures. As underlined by the aforementioned pilot studies, remote neurological examination, heart rate assessment, speech analysis, nutritional status monitoring via mHealth application, are useful and accessible tools and should be applied to all patients at the follow-up teleconsultation. While the digital evaluation of motor functions through digital devices, such as the accelerometer, are not completely reliable at the moment and should be better developed to be well tolerated by patients. A future perspective could be the development of a clinical app linked to a wearable device to achieve a complete self-assessed evaluation of nutritional status, respiratory function, motor ability, and subjectively perceived health status.

Finally, despite the high enthusiasm for telemedicine, must be kept in mind that if widespread use of telemedicine technology is to be achieved for the ALS clinical care, a well-structured multi-center telemedicine service with a high levels of expertise from clinical organization will have to be built; such expertise is still being built by most organizations. Despite these pitfalls, rapid progress is being made on several forefronts and different innovative ways forward are being developed. It is expected that continued exploration and validation of outcome measures, coupled with continued engagement of physician, nurse, speech pathologist, dietitian, respiratory therapist, engineers, biotechnologists, data scientists, physicists, and regulators, will facilitate the core goals of maximizing opportunities for patient involvement, minimizing patient and caregiver burden, and providing accurate data for analysis of outcomes.

\section{High-tech augmentative and alternative communication (HT-AAC) technologies}

The HT-AAC is a compensation form, with the aims to help and improve communication abilities of individuals with difficulties in using common channels of communication, especially verbal and written [38]. Such technologies are defined augmentative because they extend or replace means of communication for physically impaired people, but at the same time, they are defined alternative as they use multimodal methods of communication, which are different from the traditional ones, giving to patients the opportunity to maintain their communicative function by producing written or spoken messages.

Since ALS patients may completely lose the ability to articulate words and phrases (dysarthria occurs in 80-95\% of ALS patients), and have impaired limb mobility (so they may be deprived to use gesture communication), they could gain enormous benefit from the HT-AAC technologies to continue communication, despite the physical impairment that otherwise would prevent it. In particular, the development of HT-AAC, such as brain-computer interface (BCI) and eye tracking (ET), could be a useful tool to bypass the important motor difficulties present in ALS patients [39].

Additionally, HT-AAC can help maintain emotional connection within families, and support social roles, thereby decreasing the caregiver burden and increasing the patient's psychosocial well-being or quality of life.

\section{Brain-computer interface (BCI) in ALS: a P300-based case study}

The BCI is a communication system, independent of the brain's normal output pathways, that enables the generation of a control signal from brain responses such as sensorimotor rhythms and evoked potentials, thus conveying messages directly to a computer, which performs the desired action. Brain activity can be monitored by several methods as: electroencephalography (EEG), magnetoencephalography (MEG), positron emission tomography (PET), functional magnetic resonance imaging (fMRI), and functional near-infrared spectroscopy (fNIRS). However, these latter two are expensive, and they have a slow time resolution that does not allow rapid communication. While EEG has a relatively short time constant, can be operated in many environments, and requires inexpensive devices, so it is the most practical and suitable method for BCI development.

Typical BCI techniques include the use of evoked potentials (such as P300) [40] or motor imagery [41], and 
Fig. 4 Process and elements of BCI system

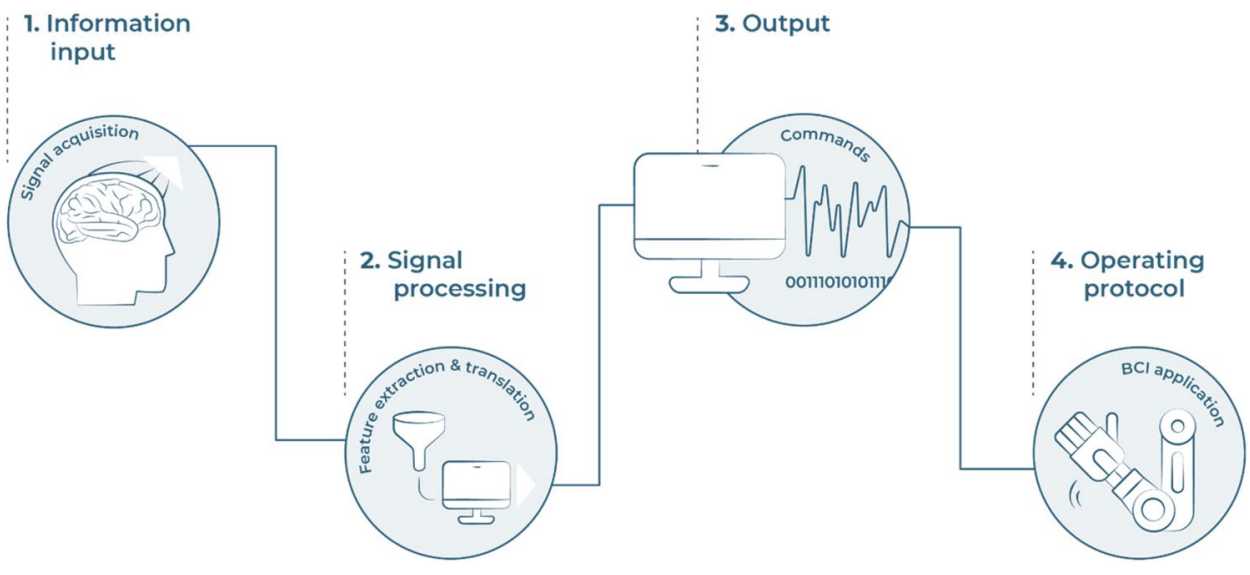

enable the user to communicate with a speller device or to control the movement of an end effector. Briefly, BCI is a communication system that does not depend on the brain's normal pathways of peripheral nerves and muscles [42, 43]. In brief, a BCI system consists of four essential parts: (1) information input (i.e. recorded brain activity from the user), (2) signal processing (i.e. the components that translate raw information into output), (3) output (i.e. the commands administered by the BCI system), and (4) operating protocol that determines the timing of operation (Fig. 4). These elements interact to produce the user's intention [44].

However, the use of BCI in ALS patients still shows some limitations, related to both technical and neuropsychological issues. In fact, the number of clinical trials on BCI is still relatively low [45-48]. Violaine Guy et al., evaluated in a clinical study the usability of P300 speller BCI on 20 patients with ALS [47]. The study consisted of two 3-block sessions (copy spelling, free spelling and free use) with the system in several modes of operation to evaluate its usability in terms of effectiveness, efficiency and satisfaction. Globally, the P300 speller BCI system was effective in that all participants successfully achieved all spelling tasks. All participants expressed satisfaction regarding comfort, ease of use and utility. Furthermore, all participants decided to try word prediction; the authors observed that the spelling rate (in symbols per minute) was improved with word prediction. For a few patients, word prediction decreased the spelling rate, which could be attributed to their difficulty in scanning the proposed words fast enough and in switching between typing strategies. In this regard, word prediction even if it brings a benefit may require some extra training to be fully effective. Despite these findings, the authors raised a number of technical and practical concerns that need to be addressed for regular daily use of the system. First, their system was designed to be set up by physicians and caregivers, so it should be further simplified to allow anyone to set it up easily. Second, the installation time of $13 \mathrm{~min}$ should
Table 2 Values of speller size parameters used by Teresa MedinaJulià et al. [49]

\begin{tabular}{lcll}
\hline Size & Speller size $(\mathrm{cm})$ & $\begin{array}{l}\text { Symbol size } \\
(\mathrm{cm})\end{array}$ & $\begin{array}{l}\text { Symbol } \\
\text { distance } \\
(\mathrm{cm})\end{array}$ \\
\hline Small & 5.27 & 0.42 & 0.55 \\
Medium & 9.98 & 0.79 & 1.04 \\
Large & 14.69 & 1.17 & 1.53 \\
\hline
\end{tabular}

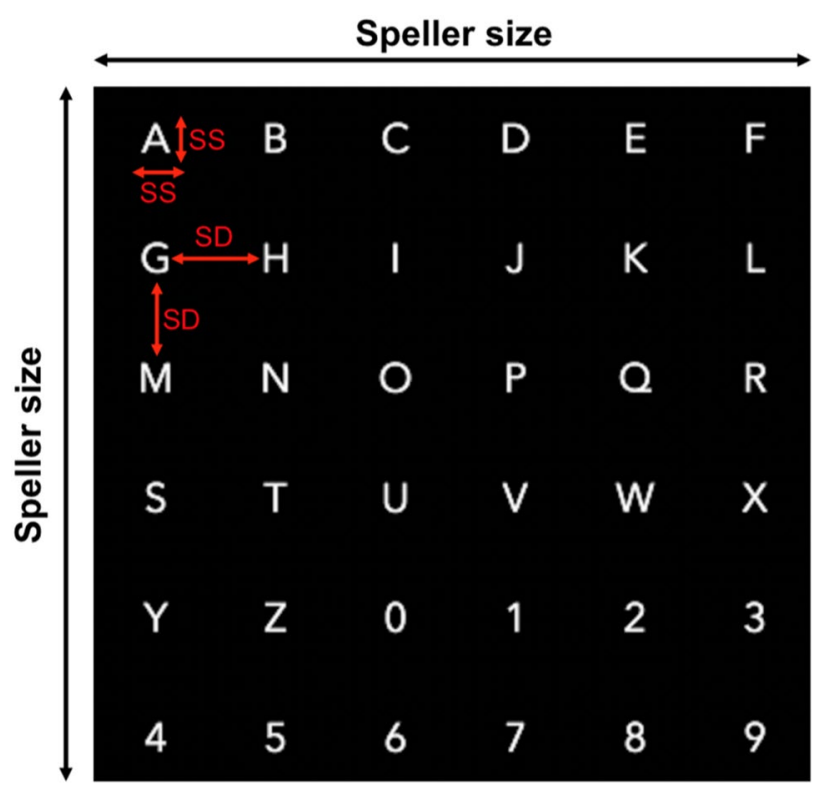

Fig. 5 Example of speller's size parameters. SS stands for "symbol size" and SD for "symbol distance"

be reduced to $<5 \mathrm{~min}$ to be practical. Finally, electrode positions should be selected to provide both good signals and good comfort even for patients who need support for holding their head (e.g. patients with the dropped head syndrome). 
Recently, Teresa Medina-Julià et al., assessed the usability of three speller sizes (Table 2, and Fig. 5) in ALS patients (mean age: $64.43 \pm 11.1$ ) [48]. This is because, the authors thought that speller size might be limiting for some lockedin state patients, where they have restricted head and eye movements [49].

In particular, the authors evaluated the effect of these three different speller sizes, in terms of effectiveness, efficiency, and satisfaction as proposed by ISO 2000, highlighting that the medium size is the most usable and the small size is the least usable in terms of satisfaction. Furthermore, the error performance evaluation showed that the medium size as the only speller that enables efficient communication according to the MEP30 criterion [50]. Finally, the medium speller was selected as the least temporal demanding and the one that required less effort to control; on the contrary the small size was selected as the most physically demanding and the one that required more effort, based on the NASATLX scores [51].

\section{Eye-tracking in ALS: assessment of cognitive functions}

In advanced ALS disease, phonatory impairment seriously hinders the patient's interpersonal communication [52-56]; for this reason ET communication devices are used to aid communication in the later stages of ALS. The main advantage of ET devices is that they are independent of the caregiver allowing social communication and interaction with the environment. As reported by Caligari et al. the most used device is the Eye Tracking Communication Device (ETCD), which is able to detect and interpret, through an infrared camera, the patient's eye movements [57]. ETCDs merely require the patient's capability of pointing and holding the gaze on various commands displayed on the monitor. The authors showed an easy and regular use of the ETCD in ALS patients with tetraplegia and anarthria, highlighting that this device is an effective option for the management of communication deficits in the advanced stage of the disease, and it would be important to provide it as standard care since improves the quality of life of the patient who is affected by the deprivation of communication. Furthermore, ET-based technology can be used for neuropsychological and cognitive assessment in non-verbal ALS patients [58-60]. For instance, Poletti et al. reported the use of an ET-based cognitive battery assessing language, attention, and social cognition abilities, showing good levels of diagnostic accuracy and usability in ALS patients [61]. The same group used an ET-based cognitive assessment to perform a verbal-motor free cognitive flexibility test (Arrows and Colors Cognitive Test) [62].

Overall, these efforts, although seminal, can provide a comprehensive assessment of cognitive functions, and the detection of cognitive impairment throughout the course of ALS disease, revealing ET technology as a useful diagnostic tool for the near future.

\section{Neuro-rehabilitation in ALS using robotics}

Robotic rehabilitation is another emerging technology that is giving its first steps in ALS, and represents a promising tool that can be used not only to assist patients but also for their assessment and training throughout the course of the disease, as it is increasingly clear that either progressive motor weakness and cognitive decline play a significant role in ALS disability [63-65]. To date, only a few robot-assisted therapies have been tested in randomized clinical trials.

A prominent example on the feasible use of robotic technology to quantify the sensory, motor and cognitive
Fig. 6 (a) KINARM exoskeleton apparatus, and (b) Armeo Power exoskeleton apparatus a

\section{Robotic exoskeleton KINARM}

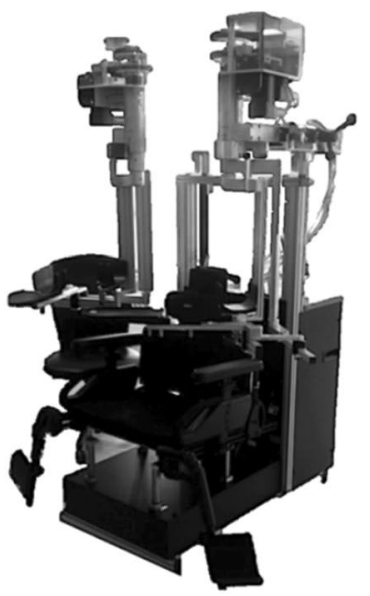

b

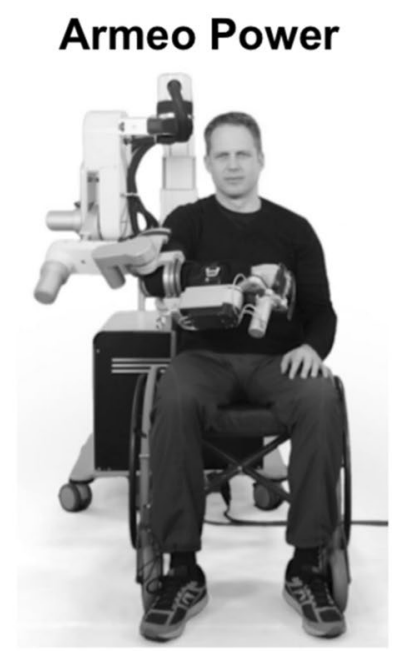


impairments of ALS patients has been reported by Leif Simmatis et al. [66]. By using the robotic exoskeleton KINARM (BKIN, Canada) (Fig. 6a) on 17 patients with ALS (mean age: $64.4 \pm 7.7$ ), the authors assessed: five tasks for upperlimb sensorimotor functions \{visually guided reaching [67], object hit [68], object hit-and-avoid [69], ball-on-bar [70], and elbow stretch test [71]\}; one task for upper limb proprioceptive function $\{$ arm position matching [72]\}; three tasks for cognition \{visually guided reaching, spatial span, and trail-making $[72,73]\}$. Most patients were able to perform the robotic tasks, showing that $56 \%$ of participants displayed motor-related impairments, $77 \%$ impairment in either arm, $69 \%$ sensorimotor impairments, and $25 \%$ proprioceptive impairments. The feasibility of using robotics (in good agreement with clinical tests, such as MoCA and FAB test), to assess motor and cognitive performance, highlight the potential of such technology in providing key additional information that can be useful for therapeutic decision-making and for clinical trials. In addition, from our point of view, it will be interesting in the future to correlate robotic motor task findings with disease stage, to have novel digital-biomarkers.

Furthermore, treatment with Armeo Power (Hocoma, Switzerland) (Fig. 6b), a robotic rehabilitation exoskeleton for upper limb training, was reported on a 69 year-old woman affected by flail arm ALS [74]. In particular, the Armeo Power is a 3D rehabilitation exoskeleton for upper limbs, allowing the treatment of motor function impairment by enhancing the residual function and neuromuscular control, assisting active movement across a large 3D workspace [75]. The Armeo Power protocol involved arm and elbow movement exercises to improve shoulder abduction, arm flexion, and elbow extension. After two months of treatment the authors observed a significant improvement in the patient's strength, together with a motor improvement of the upper limb. Although this is a single case study, this robotic neuro-rehabilitation approach with Armeo Power is a promising example of how such technology can be useful in chronic, progressive and untreatable degenerative disorders, such as flail arms ALS. Overall, robotic devices could support conventional rehabilitation, improving the patient's motor performance and enhancing plasticity phenomena within the damaged areas. However, one of the main limitations of the robotic approach is that the improvement is limited to the region of the body involved in the training, and it is difficult to imagine the combination of different robotic devices in the patient who has an impairment affecting multiple body areas.

Furthermore, it is not possible to accurately quantify the sensorimotor performance during exercise in terms of movement kinematics and exchanged forces; In the future, robotic therapies could incorporate models of the recovery process to predict the outcome of rehabilitation for adapting them to the patient's features [76, 77]. Finally, another stimulating challenge is the development of lightweight robots suitable for the domestic environments: this implies a modular structure, which facilitates donning and transportability.

In addition, it should be emphasized that people with physical disabilities, such as ALS, have classified object retrieval as a high-priority task for assistive robots [78]. Indeed, when people are unable to recover a dropped object on their own, they ask for the assistance of a caregiver; however, this requires a caregiver to be close and available and can diminish an individual's sense of independence. Hence, assistive robots could potentially help people with motor impairments retrieve dropped objects, and thereby gain greater independence. Chih-Hung King et al. reported the use of a small-scale teleoperated mobile robot named Dusty, which is capable of reliably picking up objects from the floor and delivering them to motor-impaired users [79]. The authors evaluated this robot with 20 people with ALS and the results show that they can use Dusty to robustly, safely and effectively retrieve an object from the floor. The users reported high satisfaction with Dusty and reported that the robot was significantly easier to use than their current object retrieval methods. However, future studies involving long-term real-world use would be required to assess the true potential of this emerging form of assistive technology, since during real world use, the locations of the robot, obstacles, and target objects would vary, and multiple obstacles might present greater challenges for object retrieval. Furthermore, obstacles might also obscure the user's view of the robot or target object. All of these factors could influence the time required to retrieve an object in the real world setting. In order to overcome these issues for effective use of assistive robots in a real home environment, cameras could be included on the robots to allow user-friendly use, or artificial intelligence and machine-learning based-technology could be coupled to the robot $[80,81]$.

\section{Incorporating emerging technologies in ALS clinical trials: the road ahead}

As we discussed in previous sections, clinical care for ALS patients increasingly incorporates emerging technologies to enable home-based care, improving access and quality of care in a progressively debilitated population. For this reason, we believe that today clinical investigators on ALS have the enormous opportunity to include such emerging technologies (e.g. televisits and home monitoring equipment) in trials to pave the way for an innovative trial design.

We are aware that these devices will require validation, but this great initial effort could improve: (1) the quality of the data (because allow to collect them in daily life and not just during the study visit), (2) minimize patient risk, 
(3) reduce the frequency of study visits, (4) reduce study duration, and (5) increase study safety. Furthermore, while the incorporation of novel outcome measures and bio-markers have the potential to enhance study design, innovative trial incorporating emerging technologies that enable the real-time data analysis might improve the type of therapy, route of therapy delivery, or therapeutic plan, adapting (in a patient-specific manner) the best dosage/posology during data collection, thus promoting the predictive, personalized, preventive and participatory exciting era for clinical research in ALS.

\section{Conclusions}

ALS may be considered a blueprint for neurodegenerative diseases to further develop and test emerging technologies, and the COVID-19 pandemic is squeezing up the development and implementation of such technologies in clinical practice. As it pointed out in this review, progress has already been made in the spread of telemedicine. In fact, it has been shown to be feasible and safe technology, with good compliance, and with time and cost savings for both patients/caregivers and healthcare systems. However, it will be essential the creation of a well-structured multi-center service for the bi-directional transmission of encrypted data, device synchronization, data storage and analysis, in accordance with ethical policies. On the other hand, BCI and ET expanded the field of augmentative and alternative communication in ALS but their potentialities still need to be verified so that they can become practical tools for the management of communication deficits, or diagnostic tools for the assessment of cognitive functions and the identification of cognitive impairment in the course of ALS disease. As well as robots are promising tools that can be used not only to assist patients but also for their assessment and training throughout the course of the disease. However, at the moment, they are mainly used for scientific purposes or available only to a limited number of patients, under strict protocols.

In our opinion, we have only scratched the surface of the development possibilities of these emerging technologies, and we foresee a number of great opportunities for future research in this field, in particular for the home management not only of ALS patients, but also for Parkinson's, Alzheimer's, strokes, traumatic brain injuries, and spinal cord injuries. To emphasize, nowadays, ALS is a disease that is inexorably progressive and without cure, and the gains that can be obtained with these emerging technologies can be wiped out by the subsequent loss of motor function. However, this should not be seen as a deterrent but on the contrary, the increasingly in-depth study of the mechanisms of neurodegeneration in ALS must be carried out to understand how this disease can be clinically treated. In doing so, emerging technologies such as telemedicine, assistive robotics, HT-AAC, and neural interfaces will find their deserved role in enabling patients to have a dignified life with a chronic disease.

Finally, as previously reported by Semprini et al. [63]: when developing or implementing emerging technologies targeting a specific set of populations, it is extremely necessary that patients are involved in experimental studies, right from the start; in fact, their motto is "Nothing about us, without us".

Acknowledgements We are grateful to Fondazione Serena Onlus and Gruppo Cooperativo CGM for supporting NeMO Lab research projects.

Author contributions "Conceptualization, RP; data curation, RP, BB writing — original draft preparation, RP; writing — review and editing, RP, RS, SR, BB, CL; project administration, RP. All authors have read and agreed to the published version of the manuscript.

\section{Declarations}

Conflicts of interest The authors declare no competing interest.

\section{References}

1. Masrori P, Van Damme P (2020) Amyotrophic lateral sclerosis: a clinical review. Eur J Neurol 27(10):1918-1929. https://doi.org/ 10.1111/ene. 14393

2. Zarei S, Carr K, Reiley L, Diaz K, Guerra O, Altamirano PF, Pagani W, Lodin D, Orozco G, Chinea A (2015) A comprehensive review of amyotrophic lateral sclerosis. Surg Neurol Int 6:171. https://doi.org/10.4103/2152-7806.169561

3. Nguyen C, Caga J, Mahoney CJ, Kiernan MC, Huynh W (2021) Behavioural changes predict poorer survival in amyotrophic lateral sclerosis. Brain Cogn 150:105710. https://doi.org/10.1016/j. bandc.2021.105710

4. Brown RH, Al-Chalabi A (2017) Amyotrophic lateral sclerosis. N Engl J Med 377(2):162-172. https://doi.org/10.1056/NEJMr a1603471

5. Ferrari R, Kapogiannis D, Huey ED, Momeni P (2011) FTD and ALS: a tale of two diseases. Curr Alzheimer Res 8(3):273-294. https://doi.org/10.2174/156720511795563700

6. Nitrini R (2014) Frontotemporal dementia and amyotrophic lateral sclerosis: revisiting one of the first case reports with neuropathology examination. Dement Neuropsychol 8(1):83-86. https://doi. org/10.1590/S1980-57642014DN81000013

7. Niedermeyer S, Murn M, Choi PJ (2019) Respiratory failure in amyotrophic lateral sclerosis. Chest 155(2):401

8. Cluskey S, Ramsden DB (2001) Mechanisms of neurodegeneration in amyotrophic lateral sclerosis. Mol Pathol 54(6):386-392

9. Barber SC, Mead RJ, Shaw PJ (2006) Oxidative stress in ALS a mechanism of neurodegeneration and a therapeutic target. Biochim Biophys Acta 1762(11-12):1051-1067. https://doi.org/10. 1016/j.bbadis.2006.03.008

10. Dharmadasa T, Kiernan MC (2018) Riluzole, disease stage and survival in ALS. Lancet Neurol 17(5):385-386. https://doi.org/ 10.1016/S1474-4422(18)30091-7

11. Fang T, Al Khleifat A, Meurgey JH, Jones A, Leigh PN, Bensimon G, Al-Chalabi A (2018) Stage at which riluzole treatment 
prolongs survival in patients with amyotrophic lateral sclerosis: a retrospective analysis of data from a dose-ranging study. Lancet Neurol 17(5):416-422. https://doi.org/10.1016/S1474-4422(18) 30054-1

12. Breiner A, Zinman L, Bourque PR (2020) Edaravone for amyotrophic lateral sclerosis: barriers to access and lifeboat ethics. CMAJ 192(12):E319-E320. https://doi.org/10.1503/cmaj.191236

13. Rothstein JD (2017) Edaravone: a new drug approved for ALS. Cell 171(4):725. https://doi.org/10.1016/j.cell.2017.10.011

14. Dorst J, Ludolph AC (2019) Non-invasive ventilation in amyotrophic lateral sclerosis. Ther Adv Neurol Disord 12:1756286419857040. https://doi.org/10.1177/1756286419 857040

15. Bourke SC, Gibson GJ (2004) Non-invasive ventilation in ALS: current practice and future role. Amyotroph Lateral Scler Other Motor Neuron Disord 5(2):67-71. https://doi.org/10.1080/14660 820410020330

16. Mayadev AS, Weiss MD, Distad BJ, Krivickas LS, Carter GT (2008) The amyotrophic lateral sclerosis center: a model of multidisciplinary management. Phys Med Rehabil Clin N Am 19(3):619-631. https://doi.org/10.1016/j.pmr.2008.04.004

17. Hogden A, Foley G, Henderson RD, James N, Aoun SM (2017) Amyotrophic lateral sclerosis: improving care with a multidisciplinary approach. J Multidiscip Healthc 10:205-215. https://doi. org/10.2147/JMDH.S134992

18. Hobson EV, Baird WO, Cooper CL, Mawson S, Shaw PJ, McDermott CJ (2016) Using technology to improve access to specialist care in amyotrophic lateral sclerosis: a systematic review. Amyotroph Lateral Scler Frontotemporal Degener 17(5-6):313-324. https://doi.org/10.3109/21678421.2016.1165255

19. Londral A, Pinto A, Pinto S, Azevedo L, De Carvalho M (2015) Quality of life in amyotrophic lateral sclerosis patients and caregivers: impact of assistive communication from early stages. Muscle Nerve 52(6):933-941. https://doi.org/10.1002/mus.24659

20. Pinto S, Quintarelli S, Silani V (2020) New technologies and amyotrophic lateral sclerosis - which step forward rushed by the COVID-19 pandemic? J Neurol Sci 418:117081. https://doi.org/ 10.1016/j.jns.2020.117081

21. Steinbach R, Prell T, Gaur N, Stubendorff B, Roediger A, Ilse B, Witte OW, Grosskreutz J (2020) Triage of amyotrophic lateral sclerosis patients during the COVID-19 pandemic: an application of the D50 model. J Clin Med. https://doi.org/10.3390/jcm90 92873

22. Adner R, Levinthal DA (2002) The emergence of emerging technologies. Calif Manag Rev 45(1):50

23. Day GS, Schoemaker PJH (2000) Avoiding the pitfalls of emerging technologies. Calif Manag Rev 42(2):8

24. Abercrombie RK, Udoeyop AW, Schlicher BG (2012) A study of scientometric methods to identify emerging technologies via modeling of milestones. Scientometrics 91(2):327

25. Rotolo D, Hicks D, Martin BR (2015) What is an emerging technology? Res Policy 44(10):1827

26. Moore M (1999) The evolution of telemedicine. Future Gener Comput Syst 15(2):245

27. A.A.o. Neurology, 2020. https://www.aan.com/tools-and-resou rces/practicing-neurologists-administrators/telemedicine-andremote-care/. Accessed 13 May 2021

28. Garcia-Gancedo L, Kelly ML, Lavrov A, Parr J, Hart R, Marsden R, Turner MR, Talbot K, Chiwera T, Shaw CE, Al-Chalabi A (2019) Objectively monitoring amyotrophic lateral sclerosis patient symptoms during clinical trials with sensors: observational study. JMIR Mhealth Uhealth 7(12):e13433. https://doi.org/10. $2196 / 13433$

29. Helleman J, Van Eenennaam R, Kruitwagen ET, Kruithof WJ, Slappendel MJ, Van Den Berg LH, Visser-Meily JMA, Beelen A (2020) Telehealth as part of specialized ALS care: feasibility and user experiences with "ALS home-monitoring and coaching." Amyotroph Lateral Scler Frontotemporal Degener 21(34):183-192. https://doi.org/10.1080/21678421.2020.1718712

30. Helleman J, Kruitwagen ET, van den Berg LH, Visser-Meily JMA, Beelen A (2020) The current use of telehealth in ALS care and the barriers to and facilitators of implementation: a systematic review. Amyotroph Lateral Scler Frontotemporal Degener 21(3-4):167-182. https://doi.org/10.1080/21678421. 2019.1706581

31. Vasta R, Moglia C, D’Ovidio F, Di Pede F, De Mattei F, Cabras S, Peotta L, Iazzolino B, Giusiano S, Manera U, Palumbo F, Bombaci A, Torrieri MC, Ilardi A, Mastro E, Arcari M, Solero L, Grassano M, Daviddi M, Matteoni E, Salamone P, Fuda G, Canosa A, Chio A, Calvo A (2020) Telemedicine for patients with amyotrophic lateral sclerosis during COVID-19 pandemic: an Italian ALS referral center experience. Amyotroph Lateral Scler Frontotemporal Degener. https://doi.org/10.1080/21678421.2020. 1820043

32. Bombaci A, Abbadessa G, Trojsi F, Leocani L, Bonavita S, Lavorgna L, W. Digital Technologies, N. Social Media Study Group of the Italian Society of (2021) Telemedicine for management of patients with amyotrophic lateral sclerosis through COVID-19 tail. Neurol Sci 42(1):9-13. https://doi.org/10.1007/ s10072-020-04783-x

33. Geronimo A, Wright C, Morris A, Walsh S, Snyder B, Simmons Z (2017) Incorporation of telehealth into a multidisciplinary ALS Clinic: feasibility and acceptability. Amyotroph Lateral Scler Frontotemporal Degener 18(7-8):555-561. https://doi.org/10. 1080/21678421.2017.1338298

34. Govindarajan R, Berry JD, Paganoni S, Pulley MT, Simmons Z (2020) Optimizing telemedicine to facilitate amyotrophic lateral sclerosis clinical trials. Muscle Nerve 62(3):321-326. https://doi. org/10.1002/mus.26921

35. Wills AM, Garry J, Hubbard J, Mezoian T, Breen CT, Ortiz-Miller C, Nalipinski P, Sullivan S, Berry JD, Cudkowicz M, Paganoni S, Chan J, Macklin EA (2019) Nutritional counseling with or without mobile health technology: a randomized open-label standard-ofcare-controlled trial in ALS. BMC Neurol 19(1):104. https://doi. org/10.1186/s12883-019-1330-6

36. Vitacca M, Comini L, Tentorio M, Assoni G, Trainini D, Fiorenza D, Morini R, Bruletti G, Scalvini S (2010) A pilot trial of telemedicine-assisted, integrated care for patients with advanced amyotrophic lateral sclerosis and their caregivers. J Telemed Telecare 16(2):83-88. https://doi.org/10.1258/jtt.2009.090604

37. Brearly TW, Shura RD, Martindale SL, Lazowski RA, Luxton DD, Shenal BV, Rowland JA (2017) Neuropsychological test administration by videoconference: a systematic review and metaanalysis. Neuropsychol Rev 27(2):174-186. https://doi.org/10. 1007/s11065-017-9349-1

38. Linse K, Aust E, Joos M, Hermann A (2018) Communication matters-pitfalls and promise of hightech communication devices in palliative care of severely physically disabled patients with amyotrophic lateral sclerosis. Front Neurol 9:603. https://doi.org/ 10.3389/fneur.2018.00603

39. Pasqualotto E, Matuz T, Federici S, Ruf CA, Bartl M, Olivetti Belardinelli M, Birbaumer N, Halder S (2015) Usability and workload of access technology for people with severe motor impairment: a comparison of brain-computer interfacing and eye tracking. Neurorehabil Neural Repair 29(10):950-957. https://doi. org/10.1177/1545968315575611

40. Wang C, Guan C, Zhang H (2005) P300 brain-computer interface design for communication and control applications. Conf Proc IEEE Eng Med Biol Soc 2005:5400-5403. https://doi.org/10. 1109/IEMBS.2005.1615703

41. Pfurtscheller G, Neuper C, Schlogl A, Lugger K (1998) Separability of EEG signals recorded during right and left motor imagery 
using adaptive autoregressive parameters. IEEE Trans Rehabil Eng 6(3):316-325. https://doi.org/10.1109/86.712230

42. Wolpaw JR, Birbaumer N, Heetderks WJ, McFarland DJ, Peckham PH, Schalk G, Donchin E, Quatrano LA, Robinson CJ, Vaughan TM (2000) Brain-computer interface technology: a review of the first international meeting. IEEE Trans Rehabil Eng 8(2):164-173. https://doi.org/10.1109/tre.2000.847807

43. Wolpaw JR, Birbaumer N, McFarland DJ, Pfurtscheller G, Vaughan TM (2002) Brain-computer interfaces for communication and control. Clin Neurophysiol 113(6):767-791. https://doi. org/10.1016/s1388-2457(02)00057-3

44. Cipresso P, Carelli L, Solca F, Meazzi D, Meriggi P, Poletti B, Lule D, Ludolph AC, Silani V, Riva G (2012) The use of P300based BCIs in amyotrophic lateral sclerosis: from augmentative and alternative communication to cognitive assessment. Brain Behav 2(4):479-498. https://doi.org/10.1002/brb3.57

45. Nijboer F, Sellers EW, Mellinger J, Jordan MA, Matuz T, Furdea A, Halder S, Mochty U, Krusienski DJ, Vaughan TM, Wolpaw JR, Birbaumer N, Kubler A (2008) A P300-based brain-computer interface for people with amyotrophic lateral sclerosis. Clin Neurophysiol 119(8):1909-1916. https://doi.org/10.1016/j.clinph. 2008.03.034

46. Mak JN, McFarland DJ, Vaughan TM, McCane LM, Tsui PZ, Zeitlin DJ, Sellers EW, Wolpaw JR (2012) EEG correlates of P300-based brain-computer interface (BCI) performance in people with amyotrophic lateral sclerosis. J Neural Eng 9(2):026014. https://doi.org/10.1088/1741-2560/9/2/026014

47. Guy V, Soriani MH, Bruno M, Papadopoulo T, Desnuelle C, Clerc M (2018) Brain computer interface with the P300 speller: usability for disabled people with amyotrophic lateral sclerosis. Ann Phys Rehabil Med 61(1):5-11. https://doi.org/10.1016/j.rehab. 2017.09.004

48. Medina-Julia MT, Fernandez-Rodriguez A, Velasco-Alvarez F, Ron-Angevin R (2020) P300-based brain-computer interface speller: usability evaluation of three speller sizes by severely motor-disabled patients. Front Hum Neurosci 14:583358. https:// doi.org/10.3389/fnhum.2020.583358

49. Murguialday AR, Hill J, Bensch M, Martens S, Halder S, Nijboer F, Schoelkopf B, Birbaumer N, Gharabaghi A (2011) Transition from the locked in to the completely locked-in state: a physiological analysis. Clin Neurophysiol 122(5):925-933. https://doi.org/ 10.1016/j.clinph.2010.08.019

50. Kubler A, Neumann N, Kaiser J, Kotchoubey B, Hinterberger T, Birbaumer NP (2001) Brain-computer communication: selfregulation of slow cortical potentials for verbal communication. Arch Phys Med Rehabil 82(11):1533-1539. https://doi.org/10. 1053/apmr.2001.26621

51. Lim AK, Ryu J, Yoon HM, Yang HC, Kim SK (2021) Ergonomic effects of medical augmented reality glasses in video-assisted surgery. Surg Endosc. https://doi.org/10.1007/s00464-021-08363-8

52. Ball LJ, Beukelman DR, Pattee GL (2004) Communication effectiveness of individuals with amyotrophic lateral sclerosis. J Commun Disord 37(3):197-215. https://doi.org/10.1016/j.jcomd is.2003.09.002

53. Rong P, Usler E, Rowe LM, Allison K, Woo J, El Fakhri G, Green JR (2021) Speech intelligibility loss due to amyotrophic lateral sclerosis: the effect of tongue movement reduction on vowel and consonant acoustic features. Clin Linguist Phon. https://doi.org/ 10.1080/02699206.2020.1868021

54. Roth CR, Glaze LE, Goding GS Jr, David WS (1996) Spasmodic dysphonia symptoms as initial presentation of amyotrophic lateral sclerosis. J Voice 10(4):362-367. https://doi.org/10.1016/s08921997(96)80028-2

55. Tomik B, Guiloff RJ (2010) Dysarthria in amyotrophic lateral sclerosis: a review. Amyotroph Lateral Scler 11(1-2):4-15. https://doi.org/10.3109/17482960802379004
56. Leite Neto L, Franca Junior MC, Chun RYS (2021) Speech intelligibility in people with amyotrophic lateral sclerosis (ALS). Codas 33(1):e20190214. https://doi.org/10.1590/2317-1782/ 20202019214

57. Caligari M, Godi M, Guglielmetti S, Franchignoni F, Nardone A (2013) Eye tracking communication devices in amyotrophic lateral sclerosis: impact on disability and quality of life. Amyotroph Lateral Scler Frontotemporal Degener 14(7-8):546-552. https:// doi.org/10.3109/21678421.2013.803576

58. Phukan J, Elamin M, Bede P, Jordan N, Gallagher L, Byrne S, Lynch C, Pender N, Hardiman O (2012) The syndrome of cognitive impairment in amyotrophic lateral sclerosis: a populationbased study. J Neurol Neurosurg Psychiatry 83(1):102-108. https://doi.org/10.1136/jnnp-2011-300188

59. Beeldman E, Raaphorst J, Klein Twennaar M, de Visser M, Schmand BA, de Haan RJ (2016) The cognitive profile of ALS: a systematic review and meta-analysis update. J Neurol Neurosurg Psychiatry 87(6):611-619. https://doi.org/10.1136/ jnnp-2015-310734

60. Goldstein LH, Abrahams S (2013) Changes in cognition and behaviour in amyotrophic lateral sclerosis: nature of impairment and implications for assessment. Lancet Neurol 12(4):368-380. https://doi.org/10.1016/S1474-4422(13)70026-7

61. Poletti B, Carelli L, Solca F, Lafronza A, Pedroli E, Faini A, Ticozzi N, Ciammola A, Meriggi P, Cipresso P, Lule D, Ludolph AC, Riva G, Silani V (2017) An eye-tracker controlled cognitive battery: overcoming verbal-motor limitations in ALS. J Neurol 264(6):1136-1145. https://doi.org/10.1007/s00415-017-8506-Z

62. Poletti B, Carelli L, Faini A, Solca F, Meriggi P, Lafronza A, Ciringione L, Pedroli E, Ticozzi N, Ciammola A, Cipresso P, Riva G, Silani V (2018) The arrows and colors cognitive test (ACCT): a new verbal-motor free cognitive measure for executive functions in ALS. PLoS ONE 13(8):e0200953. https://doi.org/10. 1371/journal.pone.0200953

63. Semprini M, Laffranchi M, Sanguineti V, Avanzino L, De Icco R, De Michieli L, Chiappalone M (2018) Technological approaches for neurorehabilitation: from robotic devices to brain stimulation and beyond. Front Neurol 9:212. https://doi.org/10.3389/fneur. 2018.00212

64. Braun RG, Wittenberg GF (2021) Motor recovery: how rehabilitation techniques and technologies can enhance recovery and neuroplasticity. Semin Neurol. https://doi.org/10.1055/s-0041-17251 38

65. Major ZZ, Vaida C, Major KA, Tucan P, Simori G, Banica A, Brusturean E, Burz A, Craciunas R, Ulinici I, Carbone G, Gherman B, Birlescu I, Pisla D (2020) The impact of robotic rehabilitation on the motor system in neurological diseases. A multimodal neurophysiological approach. Int J Environ Res Public Health. https://doi.org/10.3390/ijerph17186557

66. Simmatis L, Atallah G, Scott SH, Taylor S (2019) The feasibility of using robotic technology to quantify sensory, motor, and cognitive impairments associated with ALS. Amyotroph Lateral Scler Frontotemporal Degener 20(1-2):43-52. https://doi.org/10.1080/ 21678421.2018 .1550515

67. Mostafavi SM, Dukelow SP, Glasgow JI, Scott SH, Mousavi P (2014) Reduction of stroke assessment time for visually guided reaching task on KINARM exoskeleton robot. Annu Int Conf IEEE Eng Med Biol Soc 2014:5296-5299. https://doi.org/10. 1109/EMBC.2014.6944821

68. Tyryshkin K, Coderre AM, Glasgow JI, Herter TM, Bagg SD, Dukelow SP, Scott SH (2014) A robotic object hitting task to quantify sensorimotor impairments in participants with stroke. J Neuroeng Rehabil 11:47. https://doi.org/10.1186/ 1743-0003-11-47

69. Bourke TC, Lowrey CR, Dukelow SP, Bagg SD, Norman KE, Scott SH (2016) A robot-based behavioural task to quantify 
impairments in rapid motor decisions and actions after stroke. J Neuroeng Rehabil 13(1):91. https://doi.org/10.1186/ s12984-016-0201-2

70. Lowrey CR, Jackson CPT, Bagg SD, Dukelow SP, Scott SH (2014) A novel robotic task for assessing impairments in bimanual coordination post-stroke. Int J Phys Med Rehab 10(1):10

71. Centen A, Lowrey CR, Scott SH, Yeh TT, Mochizuki G (2017) KAPS (kinematic assessment of passive stretch): a tool to assess elbow flexor and extensor spasticity after stroke using a robotic exoskeleton. J Neuroeng Rehabil 14(1):59. https://doi.org/10. 1186/s12984-017-0272-8

72. Dukelow SP, Herter TM, Moore KD, Demers MJ, Glasgow JI, Bagg SD, Norman KE, Scott SH (2010) Quantitative assessment of limb position sense following stroke. Neurorehabil Neural Repair 24(2):178-187. https://doi.org/10.1177/1545968309 345267

73. Little CE, Emery C, Black A, Scott SH, Meeuwisse W, NettelAguirre A, Benson B, Dukelow S (2015) Test-retest reliability of KINARM robot sensorimotor and cognitive assessment: in pediatric ice hockey players. J Neuroeng Rehabil 12:78. https://doi. org/10.1186/s12984-015-0070-0

74. Portaro S, Cimino V, Accorinti M, Pidala A, Naro A, Calabro RS (2019) A promising tool for flail arm in amyotrophic lateral sclerosis rehabilitation: a case report. Eur J Phys Rehabil Med 55(4):515-518. https://doi.org/10.23736/S1973-9087.18.05249-8

75. Bonanno L, Russo M, Bramanti A, Calabro RS, Marino S (2019) Functional connectivity in multiple sclerosis after robotic rehabilitative treatment: a case report. Medicine (Baltimore) 98(17):e15047. https://doi.org/10.1097/MD.0000000000015047

76. Casadio M, Sanguineti V (2012) Learning, retention, and slacking: a model of the dynamics of recovery in robot therapy. IEEE Trans Neural Syst Rehabil Eng 20(3):286-296. https://doi.org/10.1109/ TNSRE.2012.2190827

77. Reinkensmeyer DJ, Burdet E, Casadio M, Krakauer JW, Kwakkel G, Lang CE, Swinnen SP, Ward NS, Schweighofer N (2016) Computational neurorehabilitation: modeling plasticity and learning to predict recovery. J Neuroeng Rehabil 13(1):42. https://doi.org/10. 1186/s12984-016-0148-3

78. Choi YS, Deyle T, Kemp CC (2009) A list of household objects for robotic retrieval prioritized by people with ALS. Robotics. arXiv:0902.2186

79. King CH, Chen TL, Fan Z, Glass JD, Kemp CC (2012) Dusty: an assistive mobile manipulator that retrieves dropped objects for people with motor impairments. Disabil Rehabil Assist Technol 7(2):168-179. https://doi.org/10.3109/17483107.2011.615374

80. Parre MD, Sujatha B (2021) Novel human-centered robotics: towards an automated process for neurorehabilitation. Neurol Res Int 2021:6690715. https://doi.org/10.1155/2021/6690715

81. Yamamoto T, Terada K, Ochiai A, Saito F, Asahara Y, Murase K (2019) Development of human support robot as the research platform of a domestic mobile manipulator. ROBOMECH J 6:3 